RESEARCH BRIEF

\title{
Onset of Regular Smoking Before Age 21 and Subsequent Nicotine Dependence and Cessation Behavior Among US Adult Smokers
}

\author{
Fatma Romeh M. Ali, $\mathrm{PhD}^{1,2}$; Israel T. Agaku, $\mathrm{PhD}^{1}$; Saida R. Sharapova, MD, MPH, CPH ${ }^{1}$; \\ Elizabeth A. Reimels, JD ${ }^{1}$; David M. Homa, PhD $^{1}$
}

Accessible Version: www.cdc.gov/pcd/issues/2020/19_0176.htm

Suggested citation for this article: Ali FRM, Agaku IT, Sharapova SR, Reimels EA, Homa DM. Onset of Regular Smoking Before Age 21 and Subsequent Nicotine Dependence and Cessation Behavior Among US Adult Smokers. Prev Chronic Dis 2020;17:190176. DOI: https://doi.org/10.5888/pcd17.190176.

\section{PEER REVIEWED}

\section{Summary}

What is already known on this topic?

Raising the minimum legal age for tobacco sales to 21 years (“T21”) is a promising strategy to reduce tobacco use among youth.

\section{What is added by this report?}

Adult smokers who started smoking regularly at age 18 to 20 years were more likely to experience high levels of nicotine dependence and less likely to attempt or intend to quit in adulthood compared with those who started at age 21 or older.

What are the implications for public health practice?

As part of a comprehensive tobacco control approach, T21 policy could help prevent lifetime addiction to nicotine and promote smoking cessation later in life.

\section{Abstract}

This study assessed the association of regular smoking initiation before age 21 years with nicotine dependence and cessation behaviors among US adult smokers. Data came from the 2014-2015 Tobacco Use Supplement to the Current Population Survey. We found that onset of regular smoking at age 18 to 20 years was associated with higher odds of nicotine dependence and lower odds of attempting and intending to quit. These outcomes were observed with regular smoking initiation at age 18 to 20 as well as before age 18, suggesting that efforts to prevent access to tobacco products before age 21 could reduce nicotine addiction and promote cessation later in life.

\section{Objective}

Tobacco use is the leading preventable cause of death and disease in the United States (1). Early onset of regular smoking exacerbates risks of smoking-related illnesses and death (2). Raising the minimum legal age for tobacco sales to 21 years ("T21") is a promising strategy to reduce smoking initiation and thus reduce regular smoking before age $21(3-5)$. However, there is limited evidence demonstrating how such policies may reduce nicotine dependence and increase smoking cessation. Previous research examined these outcomes with relation to initiation before age 17 $(6,7)$. This study examined whether delaying regular smoking until after age 21 had additional health benefits beyond those associated with delaying regular smoking until after age 18 .

\section{Methods}

Data came from the 2014-2015 Tobacco Use Supplement to the Current Population Survey (TUS-CPS), a nationally representative survey of US adults aged 18 years or older (average response rate, $54.2 \%$; methodology information is available through the US Census Bureau) (8). Analyses were restricted to 25,093 self-reported respondents: 22,070 current smokers who smoked at least 100 cigarettes during their lifetime and 3,023 recent former smokers who quit during the past year, all with information on age at onset of regular smoking and with current age 21 years or older (missing observations, $4.6 \%$ ).

Age at onset of regular smoking, which was based on responses to the question "How old were you when you first started smoking cigarettes fairly regularly?", was measured by using 3 indicators: less than 18 years, 18 to 20 years, and 21 years or older. 
Nicotine dependence was measured as low, medium, and high by using standardized definitions of the Heaviness of Smoking Index among current daily smokers (composed of time to first cigarette of the day and number of cigarettes smoked per day) $(9,10)$.

Quit attempt was defined as an affirmative response to the question, "During the past 12 months, have you stopped smoking for one day or longer because you were trying to quit smoking?" Recent former smokers also were included to capture all quit attempts. Intention to quit was defined as an affirmative response to the question, "Are you seriously considering quitting smoking within the next 6 months?"

Sociodemographic covariates were sex, current age, race/ethnicity, education, marital status, working status, and annual household income.

Percentages with 95\% confidence intervals (CIs) of age at onset of regular smoking and outcomes were reported. Association of age with nicotine dependence was assessed by using ordered logistic regression, whereas associations with quit attempt and intention to quit were assessed by using binary logistic regressions, controlling for sociodemographic covariates and state fixed effects. Analyses were conducted by using Stata version 14 (StataCorp LLC) and weighted to yield nationally representative estimates.

\section{Results}

\section{Prevalence of age at onset of regular smoking and outcomes}

Prevalence of onset of regular smoking before age 18, at age 18 to 20 , and at age 21 or older were $50.1 \%, 33.1 \%$, and $16.8 \%$, respectively (Table 1). Demographic characteristics varied significantly $(P<.05)$ across the 3 groups, with higher proportions of men and non-Hispanic whites among adults who started regular smoking before age 18 or at age 18 to 20 compared with those who started at age 21 or older. The proportion of those with a college degree or higher was lower among adult smokers who started regular smoking before age 18 compared with the other age groups.

Overall, 49.5\% made at least 1 quit attempt in the previous 12 months. Among current smokers, 46.2\% were considering quitting cigarette smoking seriously within the next 6 months. Among current daily smokers, prevalence of low, medium, and high nicotine dependence was $30.2 \%, 64.6 \%$, and $5.2 \%$, respectively.

\section{Association between age at onset of regular smoking and outcomes}

The adjusted odds of high nicotine dependence (vs combined medium and low nicotine dependence) were greater among those who started smoking regularly under age 18 than those who started at age 21 or older (adjusted odds ratio [AOR], 2.15;95\% CI, 1.92-2.39) (Table 2). Onset of regular smoking at age 18 to 20 also was associated with higher nicotine dependence in adulthood than at age 21 or older (AOR, 1.25; 95\% CI, 1.11-1.41).

Those who started smoking regularly before age 18 had smaller adjusted odds of quit attempt (AOR, 0.75; 95\% CI, 0.69-0.81) and intention to quit (AOR, 0.66; 95\% CI, 0.60-0.72) than those who started smoking at age 21 or older. Starting regular smoking at age 18 to 20 also was associated with smaller odds of quit attempt (AOR, 0.83; 95\% CI, 0.75-0.90) and intention to quit (AOR, 0.73; $95 \%$ CI, $0.66-0.81$ ) than starting at age 21 or older.

\section{Discussion}

The findings of this study suggest that starting smoking regularly at age 18 to 20 was associated with higher odds of nicotine dependence and lower odds of smoking cessation than starting at age 21 or older. These negative health consequences were observed with regular smoking initiation at age 18 to 20 as well as before age 18 . Therefore, efforts to prevent regular smoking initiation before age 21 could help prevent lifetime addiction to nicotine and promote smoking cessation later in life.

T21 policies aim to prevent youth access to tobacco products before age 21, reducing the likelihood of regular smoking initiation before this age. Although national empirical evidence about the impact of T21 is limited, evidence from local jurisdictions show that T21 is an effective strategy to limit the ability of youths to purchase tobacco products and to reduce the youth smoking rate $(4,5)$. Additionally, T21 is supported by most US adults, including 7 in 10 smokers (11).

This study has some limitations. First, all data were self-reported and may be subject to recall error. Second, the exact age when an individual became a regular smoker might not be precisely determined, as progression from experimental to regular smoking might have transitioned between various patterns of experimentation, quitting, relapses, and regular smoking. Third, age at which a respondent first smoked a cigarette is unknown and thus implications of this study for T21 policies should be interpreted with caution.

As the tobacco product landscape continues to evolve and new and emerging products enter the market, it is critical that T21 policies keep pace by including the diversity of tobacco products being sold in the United States, including e-cigarettes. It will also be important to continue to monitor future enactment of T2 1 policies at the state and local levels and track the impact on youth smoking initiation and adult smoking prevalence over time.

The opinions expressed by authors contributing to this journal do not necessarily reflect the opinions of the U.S. Department of Health and Human Services, the Public Health Service, the Centers for Disease Control and Prevention, or the authors' affiliated institutions. 


\section{Acknowledgments}

The authors have no financial disclosures or conflicts of interest to report. No copyrighted materials, surveys, instruments, or tools were used in this study.

The findings and conclusions in this report are those of the authors and do not necessarily represent the official position of the Centers for Disease Control and Prevention.

\section{Author Information}

Corresponding Author: Fatma Romeh M. Ali, Office on Smoking and Health, National Center for Chronic Disease Prevention and Health Promotion, Centers for Disease Control and Prevention, 4770 Buford Hwy, S107-7, Atlanta GA 30341. Telephone: 404468-4502. Email: fatma_romeh@feps.edu.eg

Author Affiliations: ${ }^{1}$ Office on Smoking and Health, Centers for Disease Control and Prevention, Atlanta, Georgia. ${ }^{2}$ Department of Economics, Faculty of Economics and Political Science, Cairo University, Giza, Egypt.

\section{References}

1. US Department of Health and Human Services. The health consequences of smoking -50 years of progress: a report of the Surgeon General. Atlanta (GA): Centers for Disease Control and Prevention, National Center for Chronic Disease Prevention and Health Promotion, Office on Smoking and Health; 2014.

2. Choi SH, Stommel M. Impact of age at smoking initiation on smoking-related morbidity and all-cause mortality. Am J Prev Med 2017;53(1):33-41.

3. Institute of Medicine. Public health implications of raising the minimum age of legal access to tobacco products. Washington (DC): The National Academies Press; 2015.

4. Kessel Schneider S, Buka SL, Dash K, Winickoff JP, O'Donnell L. Community reductions in youth smoking after raising the minimum tobacco sales age to 21 . Tob Control 2016;25(3):355-9.

5. Zhang X, Vuong TD, Andersen-Rodgers E, Roeseler A. Evaluation of California's 'Tobacco 21' law. Tob Control 2018;27(6):656-62.

6. Khuder SA, Dayal HH, Mutgi AB. Age at smoking onset and its effect on smoking cessation. Addict Behav 1999; 24(5):673-7.

7. Breslau N, Peterson EL. Smoking cessation in young adults: age at initiation of cigarette smoking and other suspected influences. Am J Public Health 1996;86(2):214-20.
8. US Census Bureau. Current Population Survey methodology. https://www.census.gov/programs-surveys/cps/technicaldocumentation/methodology.html. Accessed August 28, 2019.

9. Lim KH, Idzwan MF, Sumarni MG, Kee CC, Amal NM, Lim $\mathrm{KK}$, et al. Heaviness of smoking index, number of cigarettes smoked and the Fagerstrom test for nicotine dependence among adult male Malaysians. Asian Pac J Cancer Prev 2012; 13(1):343-6.

10. Chaiton MO, Cohen JE, McDonald PW, Bondy SJ. The Heaviness of Smoking Index as a predictor of smoking cessation in Canada. Addict Behav 2007;32(5):1031-42.

11. King BA, Jama AO, Marynak KL, Promoff GR. Attitudes toward raising the minimum age of sale for tobacco among U.S. adults. Am J Prev Med 2015;49(4):583-8.

\footnotetext{
The opinions expressed by authors contributing to this journal do not necessarily reflect the opinions of the U.S. Department of Health and Human Services, the Public Health Service, the Centers for Disease Control and Prevention, or the authors' affiliated institutions.
} 


\section{Tables}

Table 1. Characteristics of Adult Smokers by Age at Onset of Regular Smoking ${ }^{a}$, United States, 2014-2015

\begin{tabular}{|c|c|c|c|}
\hline Characteristics & $\begin{array}{c}\text { \% Onset of Regular Smoking at Age } \\
<18(95 \% \mathrm{Cl})\end{array}$ & $\begin{array}{c}\text { \% Onset of Regular Smoking at Age } \\
18-20(95 \% \mathrm{Cl})\end{array}$ & $\begin{array}{c}\text { \% Onset of Regular Smoking at Age } \\
\geq 21(95 \% \mathrm{Cl})\end{array}$ \\
\hline Overall & $50.1(49.6-50.6)$ & $33.1(32.6-33.6)$ & $16.8(16.4-17.1)$ \\
\hline \multicolumn{4}{|l|}{ Sex } \\
\hline Female & $43.1(42.4-43.8)$ & $44.9(44.0-45.8)$ & $50.1(48.9-51.3)$ \\
\hline Male & $56.9(56.2-57.6)$ & $55.1(54.2-56.0)$ & $49.9(48.7-51.1)$ \\
\hline \multicolumn{4}{|l|}{ Current age, $y$} \\
\hline $21-24$ & $5.6(5.2-6.0)$ & $6.7(6.1-7.3)$ & $1.8(1.4-2.3)$ \\
\hline $25-44$ & $31.6(30.9-32.3)$ & $31.3(30.4-32.1)$ & $27.5(26.4-28.6)$ \\
\hline $45-64$ & $40.6(39.9-41.3)$ & $36.2(35.4-37.1)$ & $42.3(41.1-43.5)$ \\
\hline$\geq 65$ & $22.2(21.6-22.7)$ & $25.8(25.0-26.5)$ & $28.4(27.3-29.4)$ \\
\hline \multicolumn{4}{|l|}{ Race/ethnicity } \\
\hline White, non-Hispanic & $78.5(77.8-79.1)$ & $76.7(75.9-77.5)$ & $67.7(66.5-68.9)$ \\
\hline Black, non-Hispanic & $8.0(7.6-8.4)$ & $8.5(8.0-9.0)$ & $14.0(13.1-14.8)$ \\
\hline Other, non-Hispanic & $4.4(4.1-4.8)$ & $6.0(5.5-6.5)$ & $7.5(6.7-8.2)$ \\
\hline Hispanic & $9.1(8.7-9.6)$ & $8.8(8.2-9.4)$ & $10.9(10.1-11.8)$ \\
\hline \multicolumn{4}{|l|}{ Education } \\
\hline Less than high school graduate & $15.8(15.3-16.3)$ & $8.3(7.8-8.8)$ & $10.3(9.6-11.1)$ \\
\hline High school graduate & $36.1(35.4-36.8)$ & $30.3(29.5-31.1)$ & $29.5(28.4-30.6)$ \\
\hline Some college & $31.1(30.5-31.8)$ & $33.2(32.4-34.0)$ & $32.9(31.8-34.0)$ \\
\hline College graduate or higher & $17.0(16.4-17.5)$ & $28.2(27.4-28.9)$ & $27.3(26.2-28.4)$ \\
\hline \multicolumn{4}{|l|}{ Marital status } \\
\hline Unmarried & $51.3(50.6-52.0)$ & $48.7(47.8-49.6)$ & $54.6(53.4-55.8)$ \\
\hline Married & $48.7(48.0-49.4)$ & $51.3(50.4-52.2)$ & $45.4(44.2-46.6)$ \\
\hline \multicolumn{4}{|l|}{ Working status } \\
\hline Nonworking & $45.7(45.0-46.4)$ & $41.7(40.8-42.6)$ & $45.7(44.5-46.9)$ \\
\hline Working & $54.3(53.6-55.0)$ & $58.3(57.4-59.2)$ & $54.3(53.1-55.5)$ \\
\hline \multicolumn{4}{|l|}{ Annual household income, \$ } \\
\hline$<25,000$ & $30.3(29.6-31.0)$ & $23.2(22.4-23.9)$ & $28.9(27.8-30.0)$ \\
\hline $25,000-49,999$ & $28.2(27.6-28.8)$ & $26.9(26.1-27.7)$ & $27.6(26.5-28.7)$ \\
\hline $50,000-74,999$ & $17.6(17.1-18.1)$ & $19.6(18.9-20.3)$ & $17.5(16.6-18.4)$ \\
\hline$\geq 75,000$ & $23.9(23.3-24.5)$ & $30.3(29.5-31.1)$ & $26.1(25.0-27.1)$ \\
\hline
\end{tabular}

Abbreviation: $\mathrm{Cl}$, confidence interval.

a This was defined based on respondents' answer to the survey question "How old were you when you first started smoking cigarettes fairly regularly?", categorized as $<18$ years, $18-20$ years, and $\geq 21$ years.

${ }^{b}$ Significance between subgroups was assessed by using $x^{2}$ test. Significant differences $(P<.05)$ were observed among all subgroups listed in the tables.

The opinions expressed by authors contributing to this journal do not necessarily reflect the opinions of the U.S. Department of Health and Human Services, the Public Health Service, the Centers for Disease Control and Prevention, or the authors' affiliated institutions. 
Table 2. Adjusted Odds Ratios of Nicotine Dependence and Cigarette Smoking Cessation Among US Adult Smokers, 2014-2015

\begin{tabular}{|c|c|c|c|}
\hline Characteristics & $\begin{array}{l}\text { AOR of High Nicotine Dependence }{ }^{b} \\
(95 \% \mathrm{Cl})\end{array}$ & AOR of Quit Attempt ${ }^{c}(95 \% \mathrm{Cl})$ & AOR of Intention to Quit ${ }^{\mathrm{d}}(95 \% \mathrm{Cl})$ \\
\hline \multicolumn{4}{|l|}{ Age at onset of regular smoking, $y$} \\
\hline$\geq 21$ & 1 [Reference] & 1 [Reference] & 1 [Reference] \\
\hline $18-20$ & $1.25(1.11-1.41)$ & $0.83(0.75-0.90)$ & $0.73(0.66-0.81)$ \\
\hline$<18$ & $2.15(1.92-2.39)$ & $0.75(0.69-0.81)$ & $0.66(0.60-0.72)$ \\
\hline \multicolumn{4}{|l|}{ Sex } \\
\hline Female & 1 [Reference] & 1 [Reference] & 1 [Reference] \\
\hline Male & $1.47(1.36-1.60)$ & $0.89(0.84-0.95)$ & $0.88(0.82-0.94)$ \\
\hline \multicolumn{4}{|l|}{ Current age, $y$} \\
\hline $21-24$ & 1 [Reference] & 1 [Reference] & 1 [Reference] \\
\hline $25-44$ & $1.75(1.47-2.10)$ & $0.70(0.61-0.81)$ & $1.06(0.91-1.24)$ \\
\hline $45-64$ & $2.56(2.14-3.07)$ & $0.51(0.44-0.59)$ & $0.97(0.83-1.13)$ \\
\hline$\geq 65$ & $2.14(1.74-2.64)$ & $0.45(0.38-0.53)$ & $0.68(0.57-0.82)$ \\
\hline \multicolumn{4}{|l|}{ Race/ethnicity } \\
\hline White, non-Hispanic & 1 [Reference] & 1 [Reference] & 1 [Reference] \\
\hline Black, non-Hispanic & $0.47(0.41-0.54)$ & $1.17(1.05-1.30)$ & $1.29(1.15-1.44)$ \\
\hline Other, non-Hispanic & $0.65(0.54-0.79)$ & $1.07(0.92-1.24)$ & $0.91(0.78-1.07)$ \\
\hline Hispanic & $0.29(0.24-0.34)$ & $1.17(1.04-1.33)$ & $1.11(0.97-1.27)$ \\
\hline \multicolumn{4}{|l|}{ Education } \\
\hline Less than high school graduate & 1 [Reference] & 1 [Reference] & 1 [Reference] \\
\hline High school graduate & $0.86(0.76-0.97)$ & $1.07(0.97-1.17)$ & $1.02(0.92-1.13)$ \\
\hline Some college & $0.75(0.66-0.85)$ & $1.33(1.20-1.46)$ & $1.23(1.10-1.36)$ \\
\hline College graduate or higher & $0.56(0.47-0.66)$ & $1.38(1.22-1.56)$ & $1.25(1.10-1.43)$ \\
\hline \multicolumn{4}{|l|}{ Marital status } \\
\hline Unmarried & 1 [Reference] & 1 [Reference] & 1 [Reference] \\
\hline Married & $0.90(0.83-0.98)$ & $1.11(1.04-1.19)$ & $1.06(0.99-1.14)$ \\
\hline \multicolumn{4}{|l|}{ Working status } \\
\hline Nonworking & 1 [Reference] & 1 [Reference] & 1 [Reference] \\
\hline Working & $0.84(0.77-0.92)$ & $0.90(0.84-0.97)$ & $0.95(0.88-1.02)$ \\
\hline \multicolumn{4}{|l|}{ Annual household income, \$ } \\
\hline$<25,000$ & 1 [Reference] & 1 [Reference] & 1 [Reference] \\
\hline $25,000-49,999$ & $0.87(0.79-0.97)$ & $0.99(0.91-1.07)$ & $0.93(0.85-1.01)$ \\
\hline
\end{tabular}

Abbreviations: AOR, adjusted odds ratio; $\mathrm{Cl}$, confidence interval.

a Significance was set at $P<.05$, Wald test.

${ }^{b}$ Nicotine dependence was defined for current daily smokers based on the Heaviness of Smoking Index (HSI; low, moderate, and high) (9). The HSI index was calculated from the number of cigarettes smoked per day and time to the first cigarette of the day. The model was estimated by using an ordered logistic regression model adjusted for all covariates listed in the table.

${ }^{\mathrm{C}}$ This includes both current smokers who were asked "During the past 12 months, have you stopped smoking for one day or longer because you were trying to quit smoking?" and recent former smokers who quit cigarette smoking within the previous 12 months. The model was estimated by using a binary logistic regression model adjusted for all covariates listed in the table.

d This includes current smokers who were asked "Are you seriously considering quitting smoking within the next 6 months?" The model was estimated by using a binary logistic regression model adjusted for all covariates listed in the table.

(continued on next page)

The opinions expressed by authors contributing to this journal do not necessarily reflect the opinions of the U.S. Department of Health and Human Services, the Public Health Service, the Centers for Disease Control and Prevention, or the authors' affiliated institutions. 


\section{PREVENTING CHRONIC DISEASE}

VOLUME 17, E06 PUBLIC HEALTH RESEARCH, PRACTICE, AND POLICY

(continued)

Table 2. Adjusted Odds Ratios of Nicotine Dependence and Cigarette Smoking Cessation Among US Adult Smokers, 2014-2015

\begin{tabular}{|c|c|c|c|}
\hline Characteristics & $\begin{array}{l}\text { AOR of High Nicotine Dependence } \\
(95 \% \mathrm{Cl})\end{array}$ & AOR of Quit Attempt ${ }^{\mathrm{c}}(95 \% \mathrm{Cl})$ & AOR of Intention to Quit ${ }^{d}(95 \% \mathrm{Cl})$ \\
\hline $50,000-74,999$ & $0.84(0.74-0.95)$ & $0.99(0.90-1.09)$ & $0.88(0.79-0.97)$ \\
\hline$\geq 75,000$ & $0.79(0.69-0.90)$ & $1.05(0.95-1.17)$ & $1.08(0.97-1.21)$ \\
\hline State fixed effects & Yes & Yes & Yes \\
\hline Sample size & 16,865 & 24,648 & 21,504 \\
\hline
\end{tabular}

Abbreviations: $\mathrm{AOR}$, adjusted odds ratio; $\mathrm{Cl}$, confidence interval.

${ }^{\text {a }}$ Significance was set at $P<.05$, Wald test.

${ }^{\mathrm{b}}$ Nicotine dependence was defined for current daily smokers based on the Heaviness of Smoking Index (HSI; low, moderate, and high) (9). The HSI index was calculated from the number of cigarettes smoked per day and time to the first cigarette of the day. The model was estimated by using an ordered logistic regression model adjusted for all covariates listed in the table.

${ }^{\mathrm{c}}$ This includes both current smokers who were asked "During the past 12 months, have you stopped smoking for one day or longer because you were trying to quit smoking?" and recent former smokers who quit cigarette smoking within the previous 12 months. The model was estimated by using a binary logistic regression model adjusted for all covariates listed in the table.

d This includes current smokers who were asked "Are you seriously considering quitting smoking within the next 6 months?" The model was estimated by using a binary logistic regression model adjusted for all covariates listed in the table.

The opinions expressed by authors contributing to this journal do not necessarily reflect the opinions of the U.S. Department of Health and Human Services, the Public Health Service, the Centers for Disease Control and Prevention, or the authors' affiliated institutions. 